Supplementary Materials for

\title{
Alkaline Phosphatase-Triggered Etching of Au@ FeOOH Nanoparticles for Enzyme Level Assay under Dark-Field \\ Microscopy
}

Hui Wang, ${ }^{\dagger}$ Cong-Hui Xu, ${ }^{\dagger}$ Wei Zhao* ${ }^{\dagger, \ddagger}$ Hong-Yuan Chen, ${ }^{\dagger}$ Jing-Juan Xu* ${ }^{\dagger}$

$\uparrow$ State Key Laboratory of Analytical Chemistry for Life Science, School of Chemistry and Chemical Engineering, Nanjing University, Nanjing 210023, China;

$\$$ Institute of Nanochemistry and Nanobiology, School of Environmental and Chemical Engineering, Shanghai University, Shanghai 200444, China.

*Corresponding authors. E-mail: wei_zhao@shu.edu.cn, xujj@nju.edu.cn, Tel/Fax: +86-25-89687294. 


\section{Table of contents}

S1. Experimental section.

S2. Zeta potential and DLS characterizations.

S3. TEM characterizations of Au and Au@FeOOH NPs.

S4. In-situ monitoring the growth process under DFM.

S5. XRD and XPS characterization of Au@FeOOH NPs.

S6. Contrast experiment to verify the feasibility.

S7. TEM images with the addition of different amounts of ALP.

S8. Comparison of various detection methods toward ALP level evaluation.

S9. Measurement of the ALP level in the serum sample.

S10. Reference. 


\section{S1. Experimental section.}

Instrumentation. All dark field imaging experiments were carried out on an inverted IX-73 Olympus microscope equipped with a dark field condenser $(0.8<\mathrm{NA}<0.92)$, a $60 \times$ objective lens $(\mathrm{NA}=0.7)$ and a $100 \mathrm{~W}$ halogen lamp. And dark field images were captured with a DP80 colour charged coupled device (CCD) camera (Olympus Japan). Scattering spectra were collected with a liquid nitrogen cooled electron-multiplying charged coupled device (EMCCD) camera furnished with a slit of monochromator (Acton SP2358, Princeton, USA) and grating (grating density: 300 lines $/ \mathrm{mm}$; blazed wavelength: $500 \mathrm{~nm}$ ). At the same time, the spectra of background near sample was also collected and subtracted for correction. Transmission electron microscopy (TEM), high resolution transmission microscopy (HRTEM) and high-angel annular dark-field scanning transmission microscope (HADDF-STEM) imaging were all proceeded on JEM-2100 transmission electron microscopy (JEOL Ltd., Japan) with accelerating voltage of $200 \mathrm{KV}$. Scanning electron microscope (SEM) images were acquired from Hitachi S4800 (JEOL Ltd., Japan) applied with voltages of 5-15 kV. X-ray diffraction (XRD, Bruker, D8 advance) was used to characterize the structures and components of samples with $\mathrm{Cu} \mathrm{K} \alpha$ radiation $(40 \mathrm{KV}, 40 \mathrm{~mA})$ and a scanning speed of 6 degree/min. X-ray photoelectron spectroscopy (XPS) containing survey and fine spectrum were captured from ESCALAB 250Xi (Thermo Scientific, USA). UV-Vis absorption spectroscopic characterization was acquired from a Nanodrop-2000C spectrophotometer (Thermo Fisher Scientific Inc). The hydrodynamic diameter distribution was obtained from a Brookhaven 90Plus dynamic light scattering system (Brookhaven Instruments Corporation). And the Zeta potential was measured from Zetasizer Nano-ZS system (Malvern Panalytical Ltd).

\section{S2. Zeta potential and DLS characterizations.}
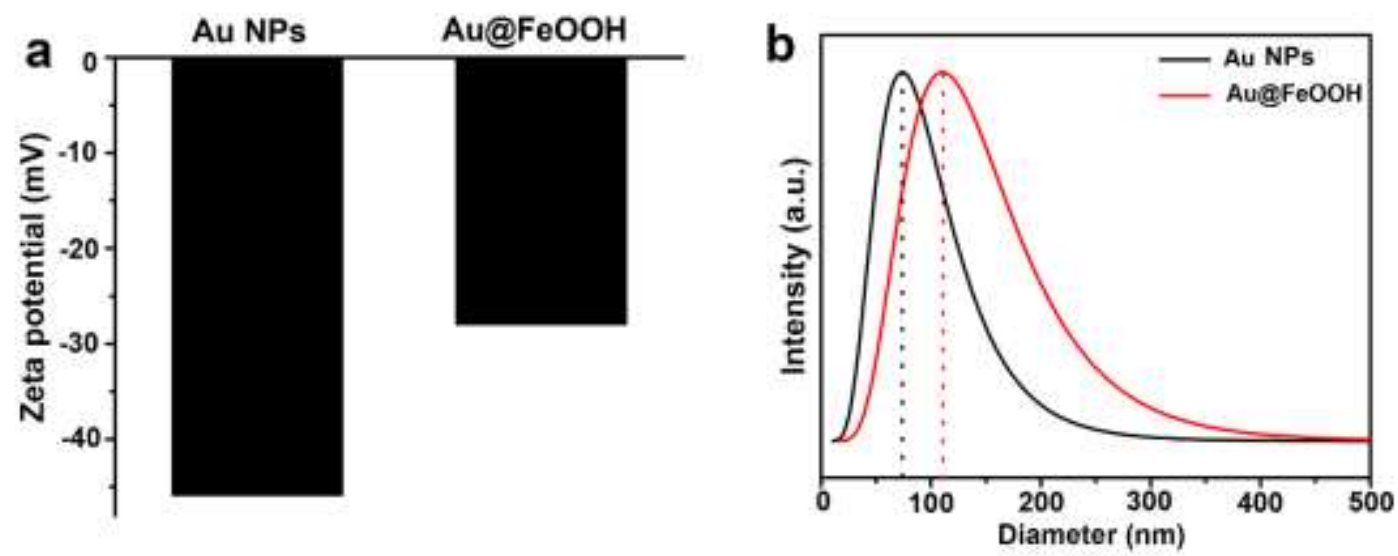

Figure S1. (a) Zeta potential and (b) DLS characterization for Au NPs and Au@FeOOH NPs, respectively. 


\section{S3. TEM characterizations of Au and Au@FeOOH NPs.}
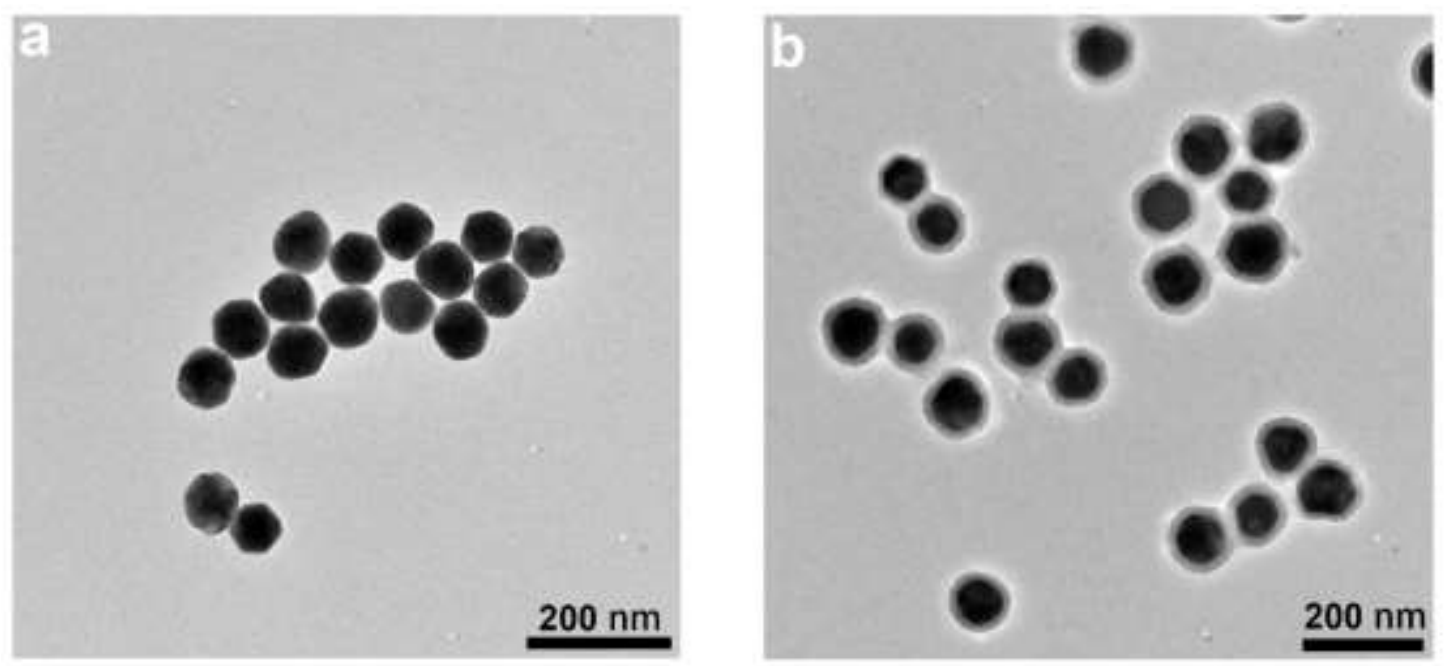

Figure S2. TEM characterizations of $\mathrm{Au}(\mathrm{a})$ and $\mathrm{Au} @ \mathrm{FeOOH}$ (b) NPs.

\section{S4. In-situ monitoring the growth process under DFM.}

As illustrated in Figure S3 and S4, as the concentration of ferrous ion continues to increase, the $\triangle \mathrm{R} / \mathrm{G}$ value also increased gradually. When the ferrous ion concentration increased to $1.0 \mathrm{mM}$, the color of these spots changed from green to orange red with R/G value increased about 0.5 in 26 $\min$ (Figure S3h). However when the ferrous ion concentration increased to $10 \mathrm{mM}$, the $\mathrm{FeOOH}$ nanostructures would formed in the aqueous solution with some bule spots come into sight of view, and the $\mathrm{R} / \mathrm{G}$ value only increased about 0.2 in $10 \mathrm{~min}$ (Figure S4c) owing to the accelerated hydrolysis rate of metal ions in aqueous solution rather than on the surface of Au NPs. Therefore, the concentration of ferrous ion was investigated to be $1.0 \mathrm{mM}$ for the optimal growth. Following, we also investigated the sodium citrate concentration during the shell formation process in Figure $\mathrm{S} 4 \mathrm{~d}$, the hydrolysis rate slowed down obviously with $0.1 \mathrm{mM}$ sodium citrate. When sodium citrate concentration increased to about $10 \mathrm{mM}$, there are many $\mathrm{FeOOH}$ nanostructures formed in the aqueous solution that would affect the DFM imaging. In conclusion, the concentration of ferrous ion and sodium citrate used for the synthesis of $\mathrm{Au} @ \mathrm{FeOOH}$ was optimized to be $1.0 \mathrm{mM}$ both. In addition to DFM imaging, the typical SEM images of several particles at different reaction stages with the optimal hydrolysis concentrations were captured in Figure S3b-f, the shell thickness gradually increased to about $20 \mathrm{~nm}$ and became uniform after the reaction duration of $26 \mathrm{~min}$. 

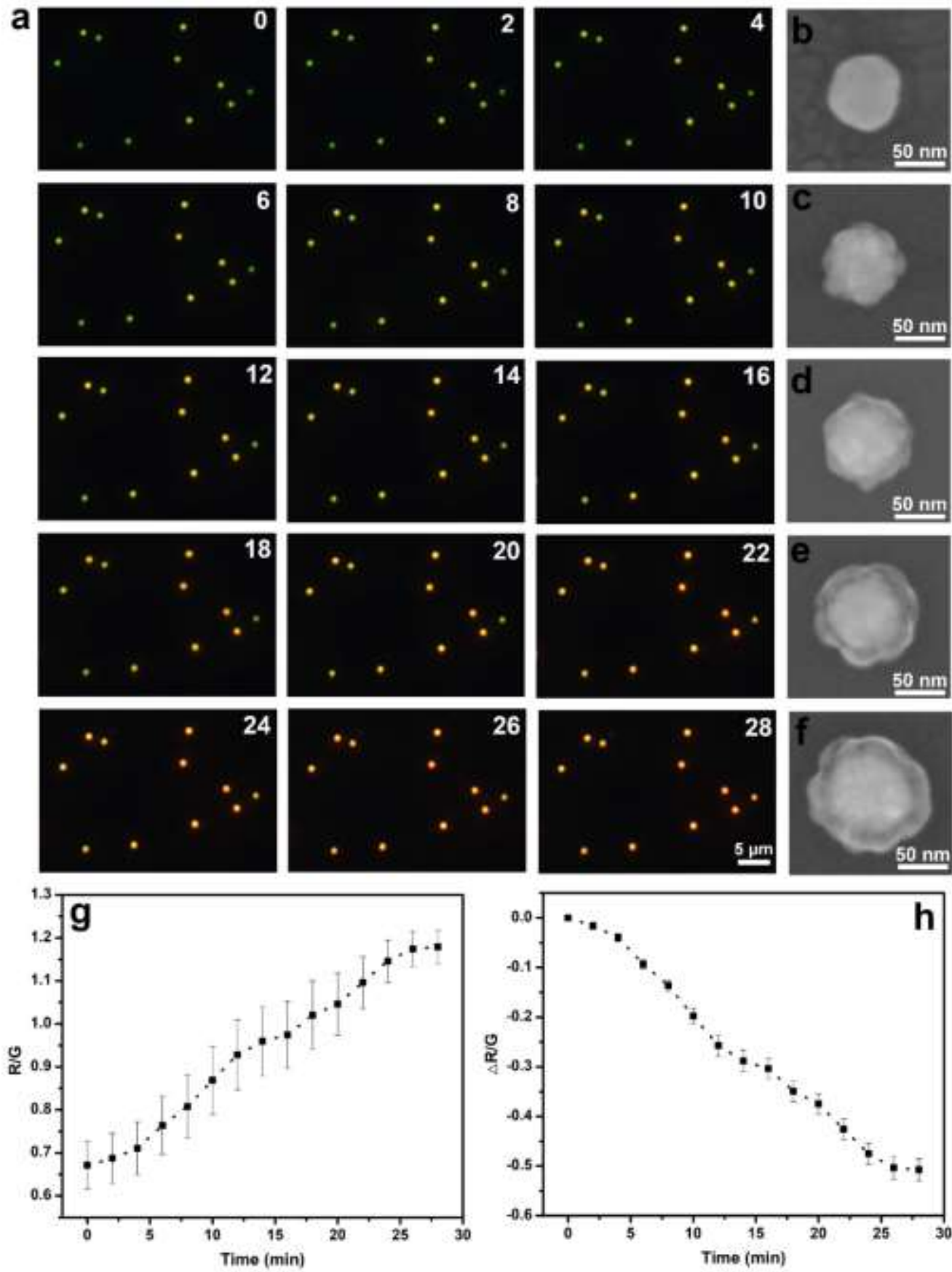

Figure S3. (a) DFM images captured during the shell growth process per 2 minute. (with $1.0 \mathrm{mM}$ sodium citrate and $1.0 \mathrm{mM}$ ferrous ion). The $\mathrm{R} / \mathrm{G}(\mathrm{g})$ and $\triangle \mathrm{R} / \mathrm{G}(\mathrm{h})$ value variations during the whole $28 \mathrm{~min}$. SEM images captured at the reaction time of $0 \min (\mathrm{b}), 8 \min (\mathrm{c}), 14 \min (\mathrm{d}), 20$ $\min (\mathrm{e})$ and $26 \min (\mathrm{f})$ 

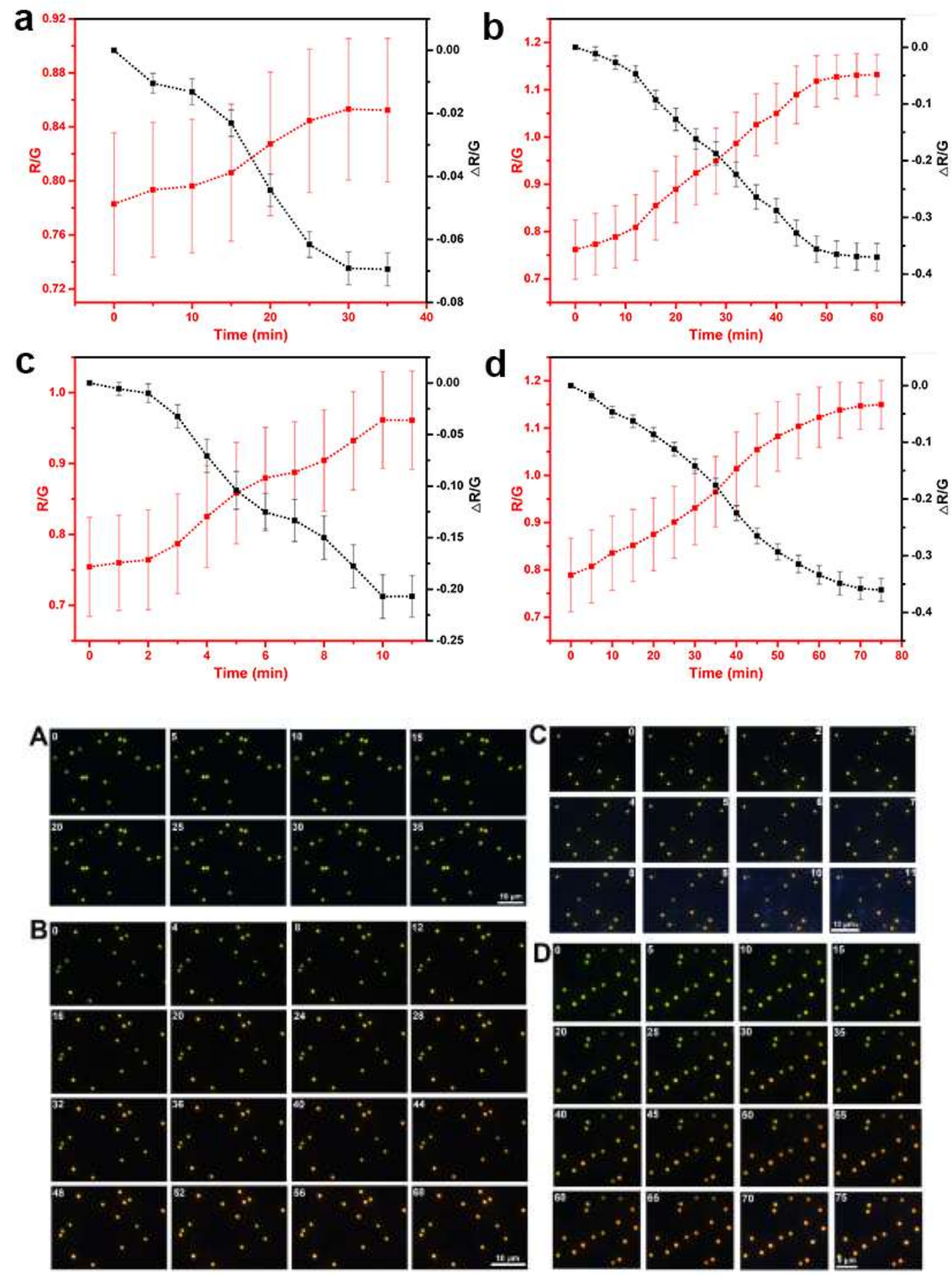

Figure $\mathrm{S} 4$. The $\mathrm{R} / \mathrm{G}, \triangle \mathrm{R} / \mathrm{G}$ variations and corresponding DFM images of various reaction solutions during the shell growth process: (a, A) $1.0 \mathrm{mM}$ sodium citrate and $0.1 \mathrm{mM}$ ferrious ion; (b, B) 1.0 $\mathrm{mM}$ sodium citrate and $0.5 \mathrm{mM}$ ferrous ion; (c,C) $1.0 \mathrm{mM}$ sodium citrate and $10 \mathrm{mM}$ ferrous ion; (d, D) $0.1 \mathrm{mM}$ sodium citrate and $1.0 \mathrm{mM}$ ferrous ion. 


\section{S5. XRD and XPS characterization of Au@FeOOH NPs.}

All these spectra were corrected on the grounds of $\mathrm{C} 1 \mathrm{~s}$ spectra. For $\mathrm{Au} 4 \mathrm{f}$ high-resolution spectrum, a doublets could be easily deconvoluted and the fitted binding energies $(87.38 \mathrm{eV}$ and $83.68 \mathrm{eV}$ ), which were attributed to $\mathrm{Au}(0) 4 \mathrm{f}_{5 / 2}$ and $4 \mathrm{f}_{7 / 2}$. The specified $\mathrm{O} 1 \mathrm{~s}$ spectrum in Figure S6e could be apparently deconvoluted into three peaks at $531.93 \mathrm{eV}, 531.13 \mathrm{eV}$ and $529.78 \mathrm{eV}$, assigned to the fitted binding energies of oxygen adsorbed on the NPs (blue line), hydroxyl in conjunction with metal ions (red line) and $\mathrm{O}^{2-}$ species (green line), respectively. Figure S6d showed the Fe $2 p$ XPS spectrum, the fitted binding energies of $724.63 \mathrm{eV}$ and $710.73 \mathrm{eV}$ were appointed as Fe $2 p 1 / 2$ and $2 p 3 / 2$, and the satellites at $718.03 \mathrm{eV}$ and $732.18 \mathrm{eV}$ were correspond to the electronic structure of $\mathrm{FeOOH} \cdot{ }^{[\mathrm{S} 1]}$

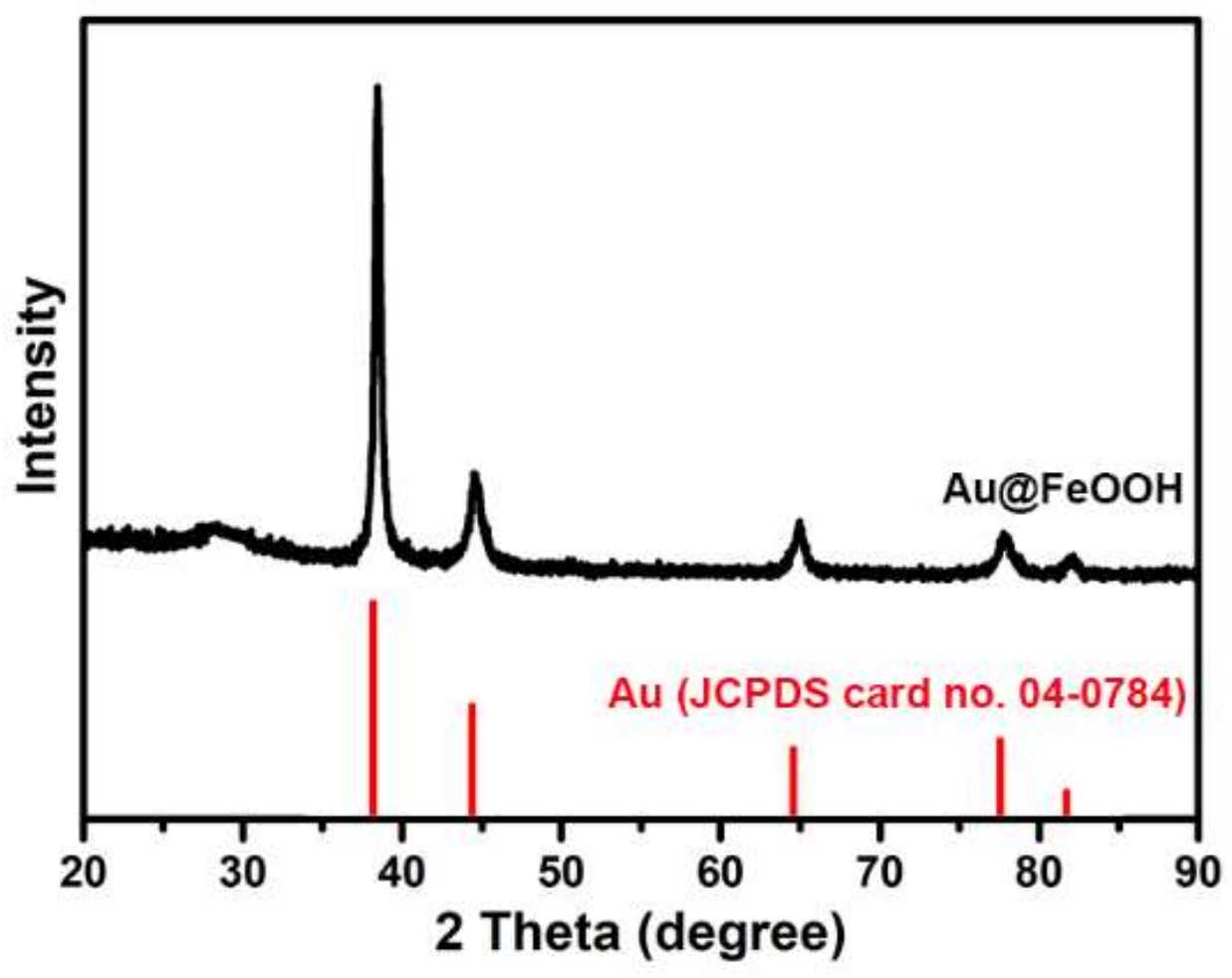

Figure S5. XRD characterization of $\mathrm{Au} @ \mathrm{FeOOH}$ NPs. 

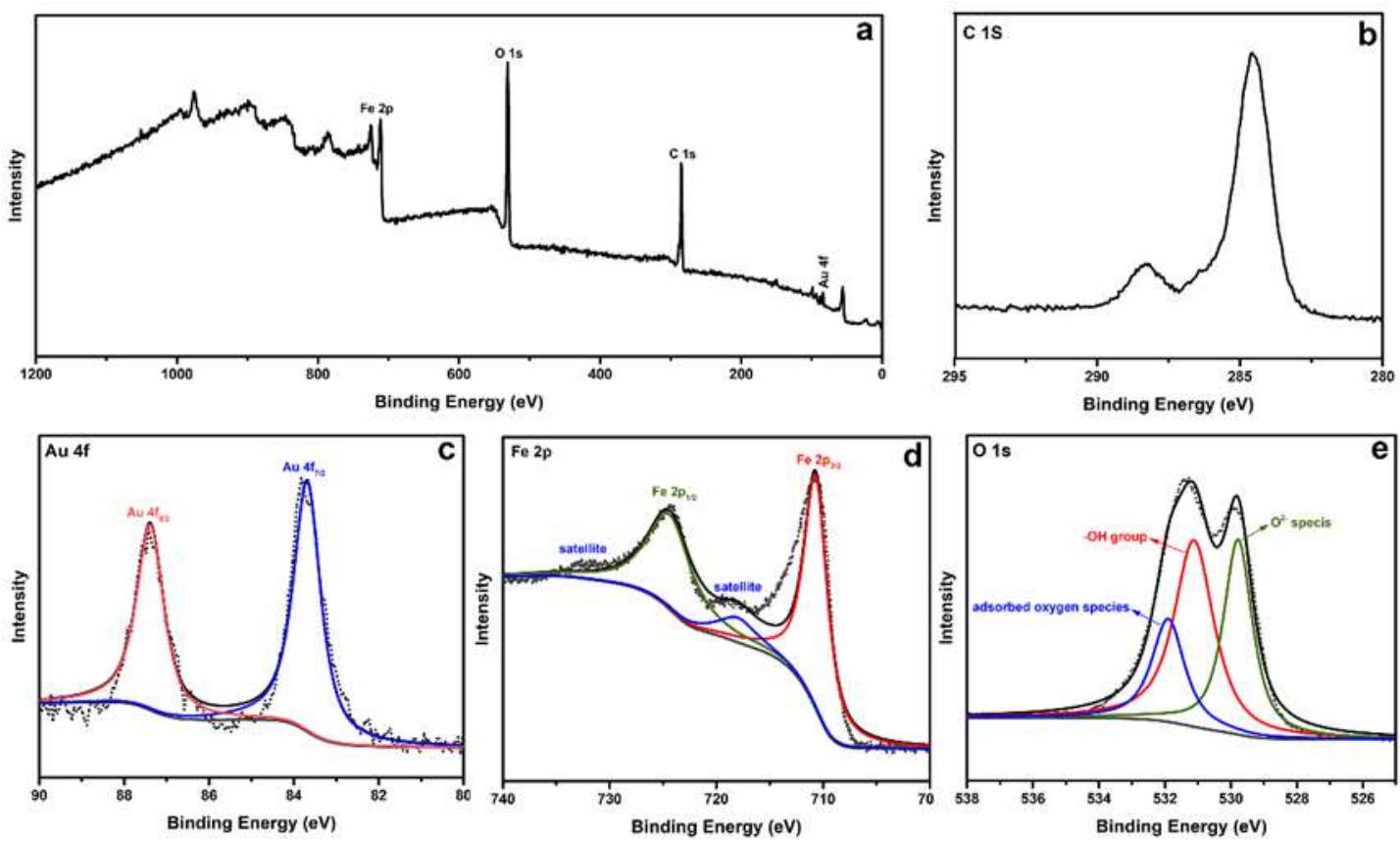

Figure S6. (a) The survey XPS spectra of Au@FeOOH NPs, (b) High resolution XPS spectra in the $\mathrm{C} 1 \mathrm{~s}$ binding energy range, (c-e) High resolution deconvoluted spectra in the Au 4f, Fe 2p and O 1s binding energy range.

\section{S6. Contrast experiment to verify the feasibility.}

The typical DFM images for Au NPs and Au@FeOOH NPs were split into three channels by Image J (red, green and blue), because the brightness of separated blue channel was negligible, The isolated red and green channels were exhibited. These channels were closely bound up to these nanoparticle's scattering spectrum, thence from distinguishing the colour of scattering spots on DFM images would be a simpler and more intuitive strategy compared to capturing the scattering spectrum through EMCCD camera and spectrometer. Statistical analysis ration of brightness for isolated red and green channel at single particle level could be used to characterize the scattering signal changes during the ALP level sensing process. The average statistical R/G values of multiple particles for Au NPs and Au@FeOOH NPs were about 0.720 and 1.169 respectively. Further, we conducted the control experiment at single particle level, Figure S7c-e showed the representative DFM images with various reaction situation. The colour (orange red) and $R / G$ value basically remain unchanged when mixed with only AA2P or ALP aqueous solution, confirming the indestructible core shell nanostructure. On the contrary, an obvious variation has been observed when the mixed aqueous solution of ALP and AA2P was added, indicating the collapse of FeOOH shell and the feasibility of this plasmonic probes toward the ALP level analysis. 

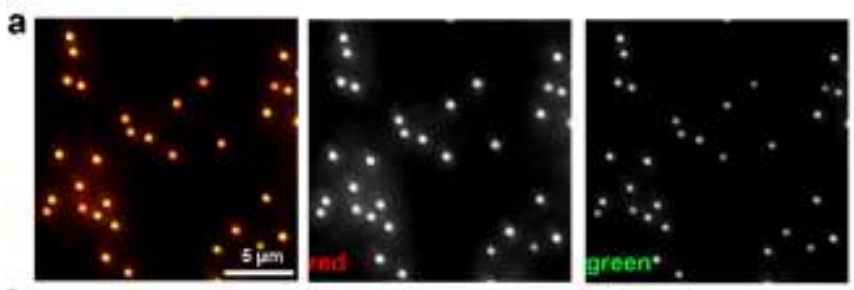

\section{Ratio \\ (red/green)}

1.169
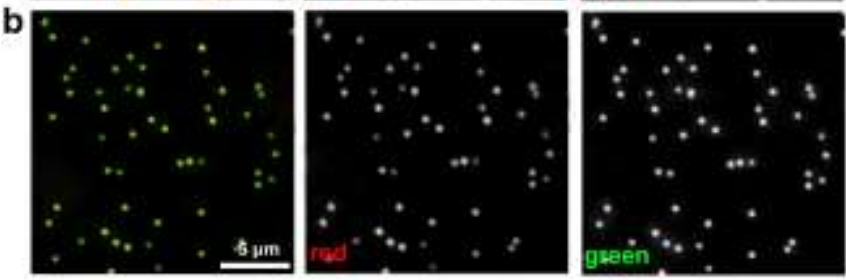

0.720

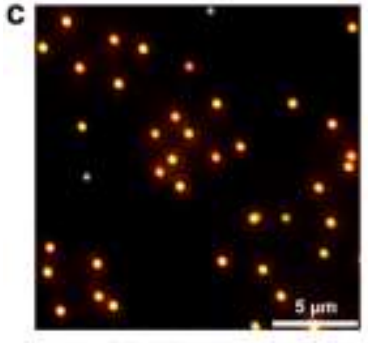

AugFeOOH NPs + ALP

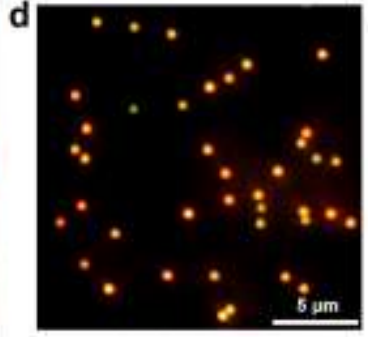

AugFeOOH NPs + AA2P

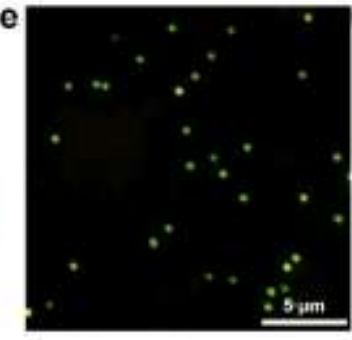

Au@FeOOH NPs + ALP + AA2P

Figure S7. DFM images of $\mathrm{Au} @ \mathrm{FeOOH}$ NPs (a) and Au NPs (b), and the corresponding images separated into red and green channels. DFM images of (c) Au@FeOOH NPs mixed with ALP aqueous solution (with ALP concentration of $20 \mathrm{U} / \mathrm{L}$ ), (d) Au@FeOOH NPs mixed with AA2P aqueous solution (with AA2P concentration of $20 \mu \mathrm{M}$ ), (e) Au@FeOOH NPs mixed with AA2P and ALP blended aqueous solution (with $20 \mu \mathrm{M} \mathrm{AA2P}$ and $20 \mathrm{U} / \mathrm{L}$ ALP).

\section{S7. TEM images with the addition of different amounts of ALP.}
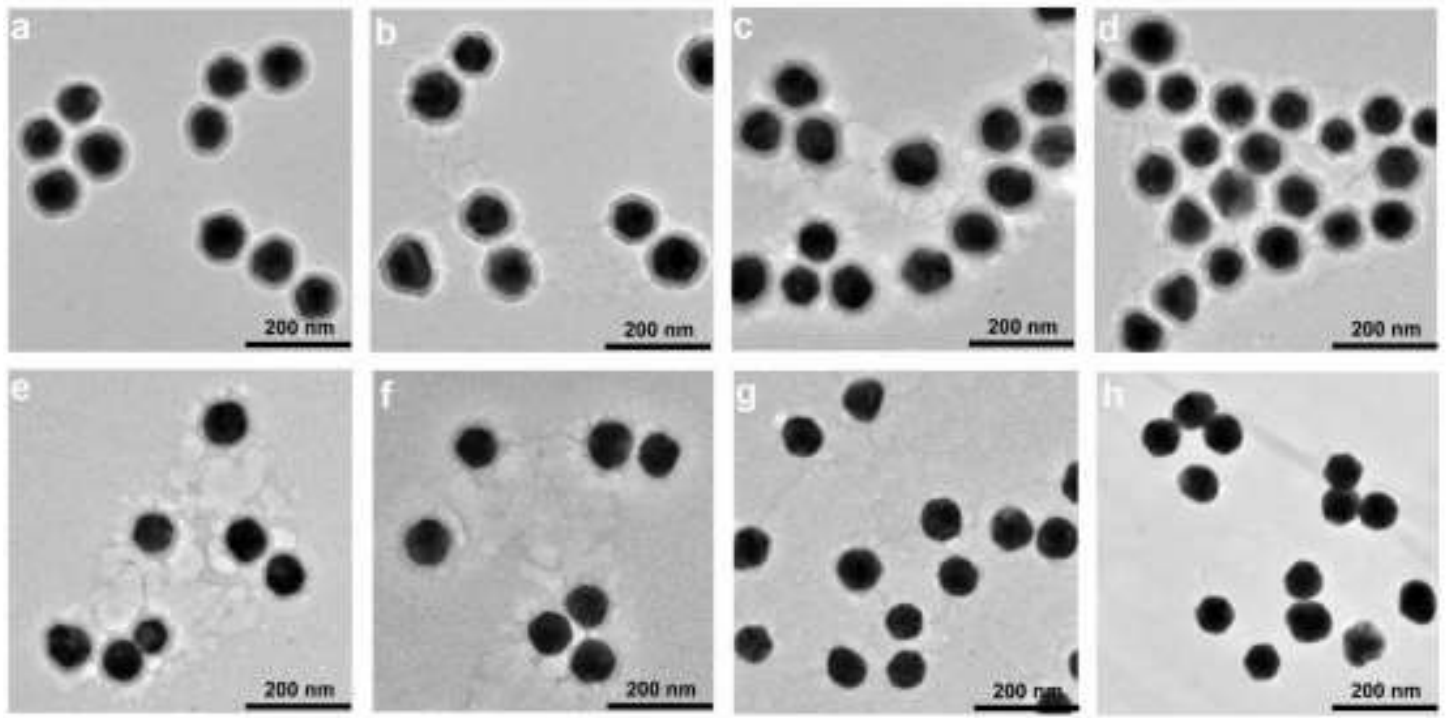

Figure S8. TEM images of $\mathrm{Au} @ \mathrm{FeOOH}$ NPs with different addition of ALP concentrations $(0,0.2$, $0.5,1.0,2.0,4.0,6.0$ and $8.0 \mathrm{U} / \mathrm{L}$ corresponding to a-h, and the AA2P concentration was fixed at $20.0 \mu \mathrm{M})$. 
S8. Comparison of various detection methods toward ALP level evaluation.

Table S1. Comparison of various detection methods toward ALP level evaluation.

\begin{tabular}{ccccc}
\hline & $\begin{array}{c}\text { Detection } \\
\text { method }\end{array}$ & $\begin{array}{c}\text { Linear range } \\
(\mathrm{U} / \mathrm{L})\end{array}$ & LOD $(\mathrm{U} / \mathrm{L})$ & Reference \\
\hline 1 & Raman & $0.4-20$ & 0.04 & $\mathrm{~S} 2$ \\
2 & Colorimetry & $2-20$ & 0.53 & $\mathrm{~S} 3$ \\
3 & Fluorescence & $1-80$ & 0.34 & $\mathrm{~S} 4$ \\
4 & Fluorescence & $2-100$ & 0.55 & $\mathrm{~S} 5$ \\
5 & Fluorescence & $1.2-14.4$ & 0.058 & $\mathrm{~S} 6$ \\
6 & Photothermal & $0.5-200$ & 0.1 & S7 \\
7 & This work & $0.2-6.0$ & 0.06 & This work \\
\hline
\end{tabular}

S9. Measurement of the ALP level in the serum sample.

Table S2. Measurement of ALP level in serum sample.

\begin{tabular}{cccc}
\hline Sample & Added (U/L) & Measured (U/L) & Recovery (\%) \\
\hline 1 & 0 & 0.48 & $/$ \\
2 & 0.5 & 1.05 & 107.1 \\
3 & 2 & 2.36 & 95.2 \\
4 & 4 & 4.30 & 96.0 \\
\hline
\end{tabular}

Note: The ALP level in the serum sample was measured to be $59 \mathrm{U} / \mathrm{L}$ by hospital, and the serum sample was 100 fold diluted in our test, the measurement result was $0.48 \mathrm{U} / \mathrm{L}$, which was close to the result that obtained from hospital.

\section{S10. Reference.}

S1. Ge, X.; Ma, Y.; Song, X.; Wang, G.; Zhang, H.; Zhang, Y.; Zhao, H., Beta-FeOOH Nanorods/Carbon Foam-Based Hierarchically Porous Monolith for Highly Effective Arsenic Removal. ACS Appl. Mater. Interfaces 2017, 9, 13480-13490.

S2. Liu, H.; Wei, L.; Hua, J.; Chen, D.; Meng, H.; Li, Z.; Xiao, L., Enzyme activity-modulated etching of gold nanobipyramids@ $\mathrm{MnO}_{2}$ nanoparticles for ALP assay using surface-enhanced Raman spectroscopy. Nanoscale 2020, 12, 10390-10398. 
S3. Zhang, Q.; Yu, Y.; Yun, X.; Luo, B.; Jiang, H.; Chen, C.; Wang, S.; Min, D., Multicolor Colorimetric Sensor for Detection of Omethoate Based on the Inhibition of the Enzyme-Induced Metallization of Gold Nanorods. ACS Applied Nano Materials 2020, 3, 5212-5219.

S4. Xiao, T.; Sun, J.; Zhao, J.; Wang, S.; Liu, G.; Yang, X., FRET Effect between Fluorescent Polydopamine Nanoparticles and $\mathrm{MnO}_{2}$ Nanosheets and Its Application for Sensitive Sensing of Alkaline Phosphatase. ACS Appl Mater Interfaces 2018, 10, 6560-6569.

S5. Li, Y.; Huang, Z. Z.; Weng, Y.; Tan, H., Pyrophosphate ion-responsive alginate hydrogel as an effective fluorescent sensing platform for alkaline phosphatase detection. Chem Commun 2019, 55, 11450-11453.

S6. He, S.-B.; Balasubramanian, P.; Hu, A.-L.; Zheng, X.-Q.; Lin, M.-T.; Xiao, M.-X.; Peng, H.-P.; Deng, H.-H.; Chen, W., One-pot cascade catalysis at neutral $\mathrm{pH}$ driven by $\mathrm{CuO}$ tandem nanozyme for ascorbic acid and alkaline phosphatase detection. Sensors and Actuators B: Chemical 2020, 321, 128511.

S7. Liu, X.; Zou, L.; Yang, X.; Wang, Q.; Zheng, Y.; Geng, X.; Liao, G.; Nie, W.; Wang, K., Pointof-Care Assay of Alkaline Phosphatase Enzymatic Activity Using a Thermometer or Temperature Discoloration Sticker as Readout. Anal Chem 2019, 91, 7943-7949. 